\title{
Electrografting of thin polymer films: Three strategies for the tailoring of functional adherent coatings
}

\author{
S. Voccia, S. Gabriel, H. Serwas, R. Jérôme, C. Jérôme
}

Center for Education and Research on Macromolecules (CERM), University of Liège, B6 Sart-Tilman, B-4000 Liège, Belgium

\begin{abstract}
Cathodic electrografting is an efficient technique to impart adhesion to poly(meth)acrylate coatings onto inorganic conducting surfaces. Although this technique was restricted for many years to very few monomers ((meth)acrylonitrile and (meth)acrylates) and to deposition of very thin polymer films, recent developments have overcome these limitations. First of all, classical controlled/living polymerization techniques have been combined with cathodic electrografting as a powerful strategy for tuning thickness, properties and reactivity of the chemisorbed organic films. Secondly, thanks to the successful electrografting of a new reactive monomer bearing an activated ester, electrografted surfaces are now available for further derivatization by a wide variety of nucleophiles. Finally, the electrografting process has been extended to the direct electrografting of reactive polymers, i.e. preformed polymers bearing pendant acrylic functions, which opens the way to the grafting of, e.g., polycondensates.
\end{abstract}

Keywords: Electrografting; Polymer films; Polycondensates

\section{Introduction}

Nowadays, the surface of a variety of inorganic substrates is coated by synthetic polymers in order to confer them specific properties (e.g., adhesion, hydrophilicity or hydrophobicity, low friction, resistance to environmental attack, biocompatibility, etc.). The major problem to be tackled is however the usually weak and short-term adhesion between organic polymers and inorganic substrates (e.g., metals), materials of a completely different nature. Covalent bonding at the interface is then by far the most desirable situation [1,2].

Electrografting of polyacrylate chains onto electrochemically conducting surfaces has proved efficiency for the deposition of very thin polymer films and the chemisorption of the constitutive chains to the surface [3-5]. Whenever acrylic monomers are polymerized under an appropriate cathodic potential in a proper organic solvent, the growing chains are chemisorbed onto the cathode even in a good solvent for them [6], which accounts for the terminology of electrografting. Because of the very fast passivation of the cathode by the polyacrylate layer and the early termination of the polymerization, the film thickness does not exceed $100 \mathrm{~nm}$. In addition, this process is mainly restricted to (meth)acrylic derivatives.

We present here some recent developments toovercome these limitations of the electrografting technique. Three strategies have been followed to reach this goal.

1) First, the electrografting has been combined with the so-called "grafting from" technique, by using, for electrograft ing, a novel inimer (Scheme 1).An inimer is a compound that contains a monomer and an initiator in the same molecule. In this work, the polymerizable part is an acrylate function and the initiator of controlled/living polymerization of (non) (meth)acrylate monomers is part of the ester group. So after the electrografting of the polyacrylate, a second family of chains can be initiated and grown from the strongly adhering polyacrylate film as shown on Scheme 1.

2) The "grafting to" technique easily follows the electrografting step since a reactive coating towards nucleophiles is prepared thanks to the use of an activated acrylic derivative for the electrochemical step. This second strategy is schematized on Scheme 2.

3) In a third strategy, preformed reactive polymers have been first synthesized by controlled/living polymerization, so that their macromolecular parameters and composition can be finely tuned. They are then electrografted in a second step by application of the required cathodic potential to a dimethyl-formamide (DMF) solution of the acrylic functionalized polymer (Scheme 3 ). 


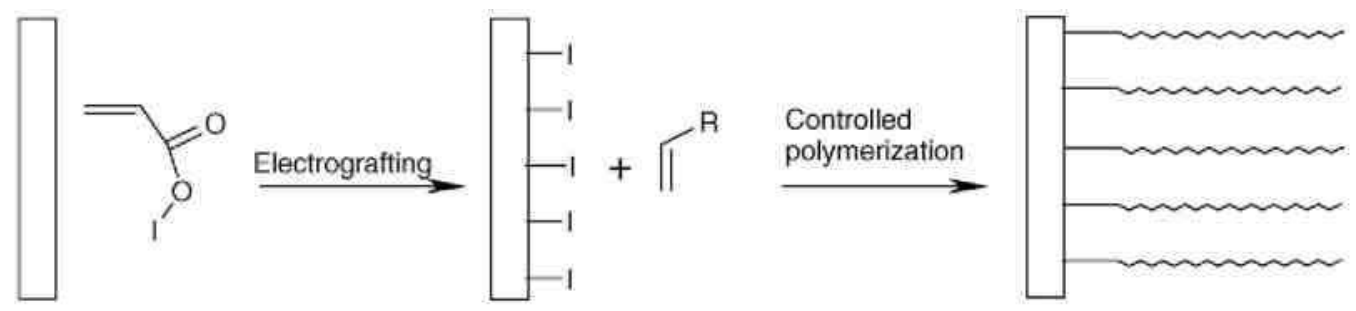

Scheme 1. "Grafting from" approach.

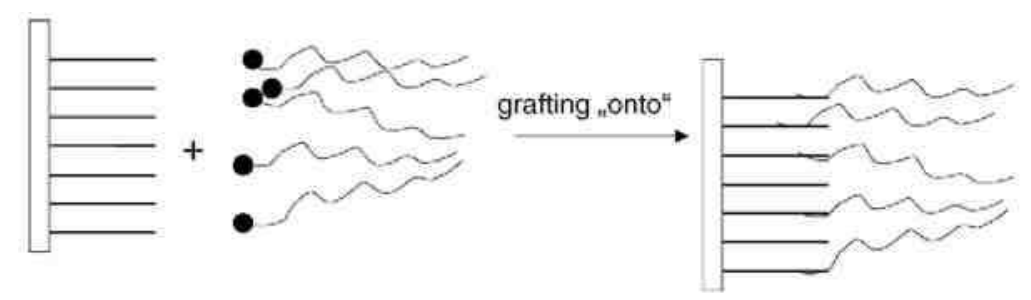

Scheme 2. "Grafting onto” approach.

All of these strategies are expected to broaden the coatings properties and afford the precise tuning of their characteristics. This is examplified in the following.

\section{Experimental}

\subsection{Materials}

All solvents and monomers were dried and distilled under reduced pressure before use. Tetraethylammonium perchlorate (TEAP, Fluka) was heated in vacuo at $80{ }^{\circ} \mathrm{C}$ for $12 \mathrm{~h}$ prior to use. Macromonomers (Scheme 6) were provided by Acros (PEO-A Mn 454 and A-PEO-AMn 258 and 700) and ABCR (A-PDMS-A, DP 18) and were dried by toluene azeotropic distillation before use.

\subsection{Electropolymerization conditions}

The acrylic monomer (or reactive polymer) was dissolved in DMF $(0.1-1.5 \mathrm{M})$ containing TEAP as a conducting salt $(0.05 \mathrm{M})$, in a classical three-electrode cell. Platinum foils were used as a counter-electrode and pseudo-reference electrode, respectively. Substrates used as working electrode (C, Fe, stainless steel, etc.) were carefully washed with heptane and acetone, and dried overnight in vacuo at $150^{\circ} \mathrm{C}$. Superficial residual oxide, if any, were removed electrochemically prior to use by repeated scanning in acetonitrile (ACN) solution of TEAP $(0.05 \mathrm{M})$. The electrografting was then performed by scanning cathodically the potential to the top of the first reductionpeak and holding it for a few seconds, i.e. until the currentfalls to a residual value. The modified substrates were then carefully washed with pure DMF in order to get rid of the unreacted monomer and any soluble ungrafted polymer chains. DMF was further removed by washing with acetonitrile. Electrochemical experiments were carried out in a glove box under inert and dry atmosphere (N2) with a PAR-EG\&G (model 273A) potensiostat/galvanostat.

\subsection{NMP "grafting from" conditions}

The electrografted carbon or stainless steel plates with inimer (Scheme 4(1)) were immersed into styrene (5 ml) added with free alkoxyamine (2-phenyl-2-(2,2,6,6-tetramethyl-piperidin-1-yloxy)-ethylbenzoate, PTEMPO) and toluene $(2 \mathrm{ml})$. The mixture is then heated under inert atmosphere at $125{ }^{\circ} \mathrm{C}$ under stirring during $18 \mathrm{~h}$. The polystyrene (PS) formed in solution was recovered by reprecipitation. The modified electrodes were cleaned using THF and dried before surface analysis.

The diblock copolymers were prepared by the same method: $n$-butylacrylate containing the 2,2,5-trimethyl-3-(1'phenylethoxy)-4-phenyl-3-azahexane alkoxyamine and the parent nitroxide were heated at $125{ }^{\circ} \mathrm{C}$ for $24 \mathrm{~h}$ in presence of the electrografted substrate. After careful washing by toluene, the modified carbon or stainless steel substrates have been dipped in styrene added with the PTEMPO alkoxyamine and heated at $125{ }^{\circ} \mathrm{C}$ for $18 \mathrm{~h}$. 


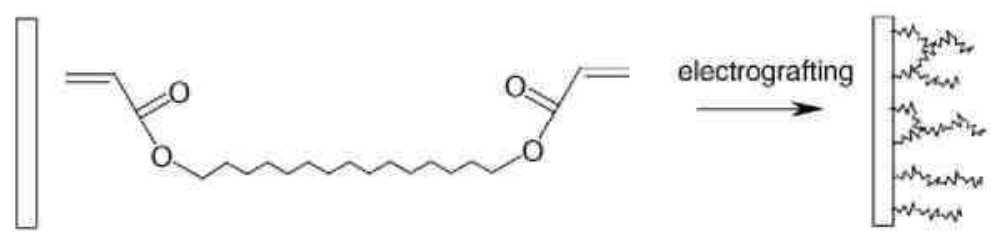

Scheme 3. "Preformed reactive polymers" approach.<smiles>C=CC(=O)OCC(ON1C(C)(C)CCCC1(C)C)c1ccccc1</smiles>

(1)<smiles>C=CC(=O)OCCOC(=O)C(C)Cl</smiles>

(2)

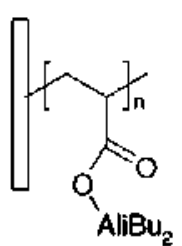

(3)<smiles>C=CCC(C)(C)C(=O)OC=C(C)C</smiles>

(4)

Scheme 4. Different inimers used in the first approach.

\subsection{Characterization techniques}

Raman spectroscopy: Dylor spectrometer, Superlabram type equipped with an 800-2000 CCD detector cooled by liquid nitrogen and a microscope. X-ray photoelectron spectroscopy (XPS): VG-Escalab 220iXL spectrometer with the monochromatized $\mathrm{Al} \mathrm{K} \alpha$ radiation at $1486.6 \mathrm{eV}$. Attenuated total reflection Fourier transformed infra-red (ATR-FTIR): Nicolet spectrophotometer. Size-exclusion chromatography (SEC): Hewlett Packard 1090 liquid chromatograph equipped with a 1037 A refractive index detector.

\section{Results and discussion}

\subsection{Combination of electro grafting with the "grafting from" technique}

Various inimers have been synthesized for electrografting and immobilization of an initiator of controlled/living polymerization by various mechanisms such as ring-opening polymerisation (ROP) [7-9] or controlled radical polymerisation $[10,11]$. By this way, the chemical properties of the organic coating is expected to be extensively tunable and the film thickness increased at will because the second polymerization step is controlled/living while, last but not least, the adhesion would be preserved. Scheme 4 shows the different inimers synthesized to initiate (1) nitroxide mediated polymerization (NMP) [11] and (2) atom transfer radical polymerization (ATRP) [10]. Similarly, ring-opening polymerization [9] of lactones and lactides can be initiated from (3), achieved by first electrografting of ethyl acrylate followed by the reduction of the poly(ethylacrylate) with diisobutylaluminum hydride. Ring-opening metathesis polymerization (ROMP) [7,8] is initiated after derivatization of poly(allylmethacrylate) with the Grubbs catalyst (4).

The inimer in Scheme 4(1) associates an (electro)polymerizable acrylate and an alkoxyamine, which is the precursor of a pair of initiator/nitroxide mediator for controlled radical polymerization. When dissolved in $\mathrm{DMF} / \mathrm{TEAP}$ solution, the recorded voltammogram onto glassy carbon is shown in Fig. 1. Clearly, a reduction peak corresponding to the grafting of this inimer is observed during the first scan. The potential is hold at the maximum of peak I, the cathode is rapidly passivated which is confirmed by the intensity of peak I, that dramatically decreases when the potential scanning is repeated until the potential of peak I (Fig. 1b). This passivation is the signature of an insulating polyacrylate film deposited on the cathode surface. 


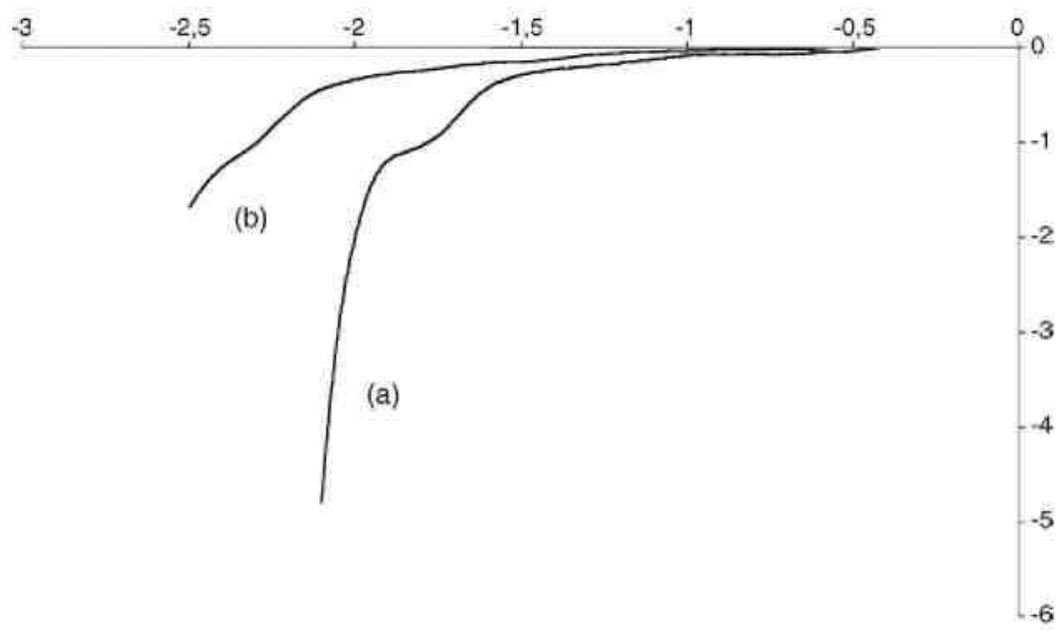

Fig. 1. Voltammogram recorded onto vitreous carbon for the electrografting of (Scheme 4(1)) (0.5 M) in DMF containing TEAP (0.05 M), scan rate $20 \mathrm{mV} / \mathrm{s}$ : (a) first scan and (b) second scan.

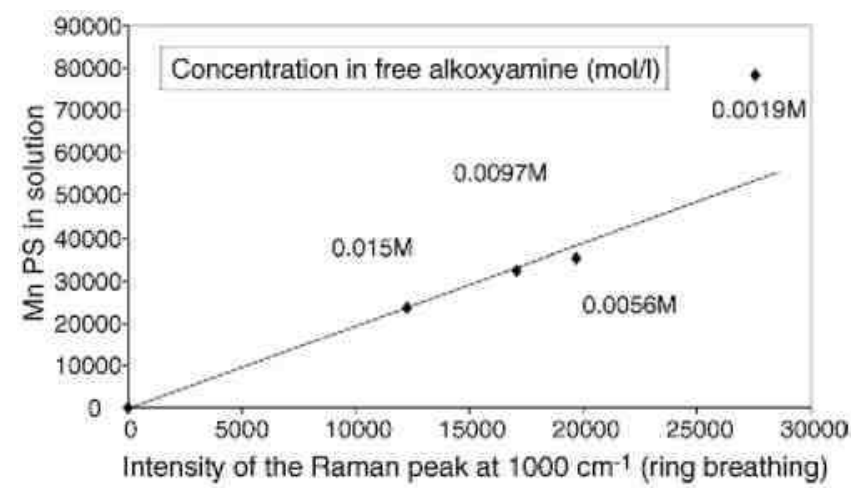

Fig. 2. Plot of the Raman signal intensity at $1000 \mathrm{~cm}^{-1}$ of polystyrene modified plates vs. Mn of ungrafted PS recovered in the solution of the same experiment for experiments performed with various amounts of free alkoxyamine in the polymerization medium.

The electrochemical stability of the alkoxyamine (particularly of the $\mathrm{C}-\mathrm{O}-\mathrm{N}$ bond of the alkoxyamine) has been ascertained by the cyclic voltammetry of a non polymerizable model for the inimer in Scheme4(1), i.e. 2-phenyl2-(2,2,6,6-tetramethyl-piperidin-1-yloxy)-ethylbenzoate (BTE), dissolved in DMF (0.1 M) together with TEAP $(0.05 \mathrm{M})$. No reaction is observed in the potential range from 0 to $-2.5 \mathrm{~V}$. Similar electrochemical behavior have also been observed when carbon was substituted for stainless steel substrates.

In order to reach grafted polystyrene chains of various molecular weights, styrene polymerization has been initiated from the chemisorbed polyacrylate chains, by immersing the modified electrode in styrene containing various amounts of free alkoxyamine (2-phenyl-2-(2,2,6,6-tetramethyl-piperidin-1-yloxy)-ethylbenzoate, PTEMPO). Upon heating, the chains are both initiated and grown in solution and from the surface. SEC analysis of the chains collected in solution have increasing molecular weight whenever the free alkoxyamine concentration is decreased in solution, as expected for a controlled process. In parallel, the Raman spectra recorded on the modified electrodes show the typical signature of polystyrene (strong band at $1100 \mathrm{~cm}^{-1}$ ) for each sample, the band being more intense when the alkoxyamine content was low in solution. Since the intensity of the Raman signal can be related to the film thickness, Fig. 2 clearly shows that the thickness of the polystyrene coating is controlled by the molecular weight of the chains, i.e. the amount of free alkoxyamine added to the styrene solution.

Because the chains initiated by NMP retain their capacity of growing for a long time, block copolymers can be grafted from the alkoxyamine containing chemisorbed polyacrylate layer by sequential polymerization of $n$ butylacrylate $(n \mathrm{BuA})$ followed by styrene. The diblock copolymer chains tethered at the surface is then able to nanostructure the surface when immersed in a solvent selective for one block. Indeed, when immersed in THF, a good solvent for both blocks, no specific structuration of the coating is observed by AFM (Fig. 3a).

Nevertheless, when the film is immersed in $n$-butanol, a selective solvent for the $n$-butylacrylate block, the nanostructuration of the surface is quite obvious (Fig. 3b) and results from the selective swelling of the PnBuA nanodomains and precipitation of the polystyrene one. 


\subsection{Combination of electrografting with the "grafting to" technique}

Succinimidyl acrylate (NSA) is an easily prepared monomer whichiswell-suitedtothe surface functionalization of an electrically conducting substrate by succinimidyl groups, which have high propensity to react with nucleophiles, such as amines [12] (Scheme 5).

Electroreduction of this monomer in a DMF/TEAP solution, gives the typical signature for electrografting,asdescribed above for the inimer. After electroreduction, the presence of the PNSA thin film on the electrode was assessed by X-ray photoelectron spectroscopy (Fig. 4) and ATR-FTIR (Fig. 7). Indeed, the analysis of the XPS C1s area shows that except for one non-attributed peak at $287 \mathrm{eV}$, all the specific carbon expected for the PNSA structure have been detected on the surface. This analysis evidences that the activated ester is not affected by the electroreduction process and that the succinimidyl groups are still available for further reactions.

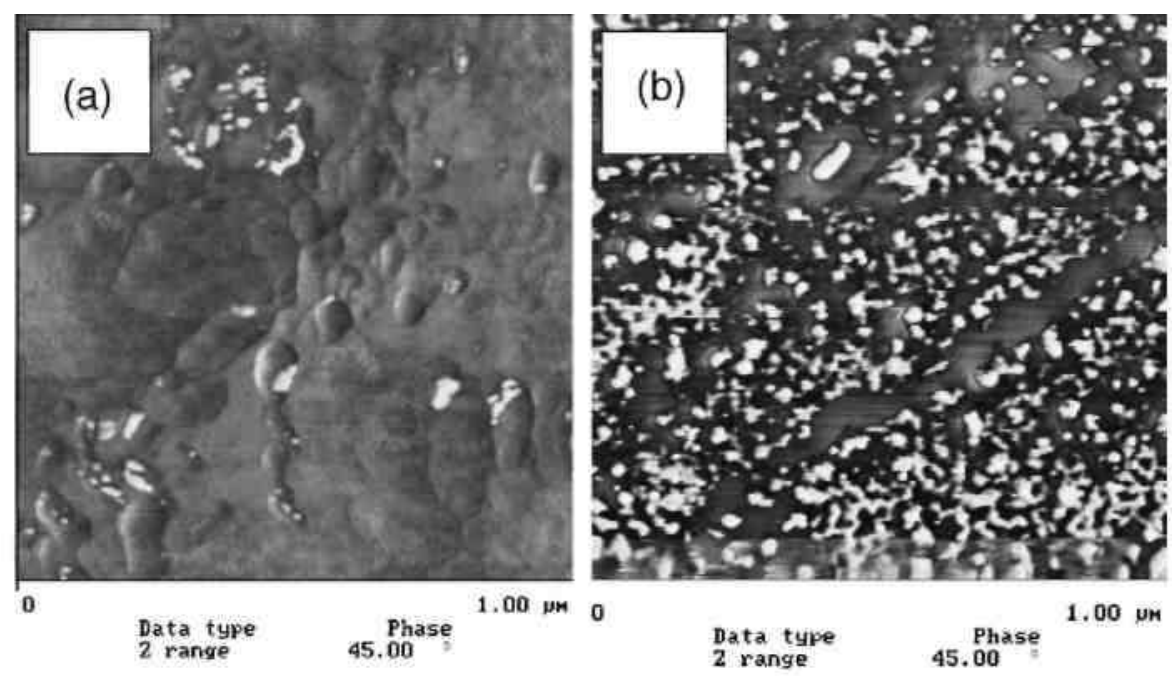

Fig. 3. AFM-images (phase contrast) of PnBuA-b-PS chains tethered at a stainless steel surface: (a) from THF and (b) from n-butanol.

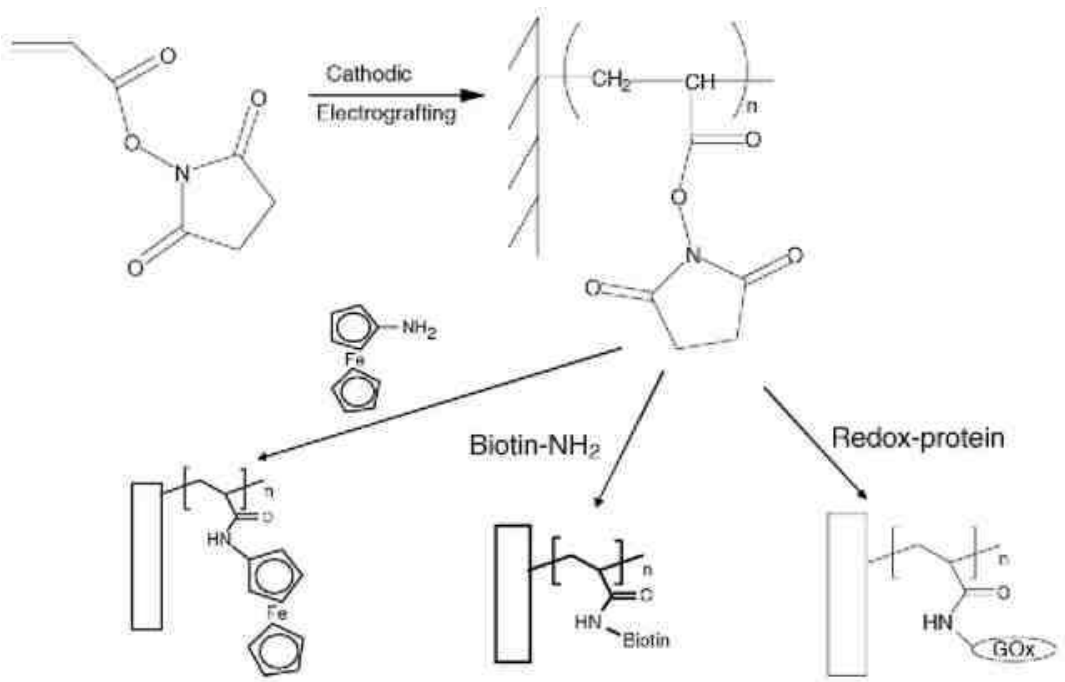

Scheme 5. Surface functionalization of PNSA by nucleophiles such as amines.

Similarly, ATR-FTIR spectrum (Fig. 7) also confirms the presence of the PNSA coating at the surface of the reflecting metallic substrate (Particularly, the $\mathrm{C}=\mathrm{O}$ ester at $1735 \mathrm{~cm}^{-1}$ ).

The succinimidyl pendant groups of the chemisorbed polyacrylate chains are thus valuable anchoring sites for any type of amine containing (macro)molecules as evidenced by using aminoferrocene as a model molecule. Reaction of the PNSA coating with aminoferrocene results indeedinelectroactive coatings (Fig. 5).

The strongly adhering PNSA filmisalso a valuable primer for the anchoring of a wide range of amine containing 
molecules of biological interest, such as biotin, enzymes and redox proteins. It is an ideal platform for the design of enzymes biosensors.

\subsection{Electrografting of preformed reactive polymers}

The last strategy consists in using polymers bearing acrylic functions for the direct electrografting on the conducting material. This strategy already reported for reactive polyesters bearing pendant acrylic functions along the entire backbone [13], has been tested here for a series of commercially available acrylic end-capped macromonomers listed in Scheme 6.

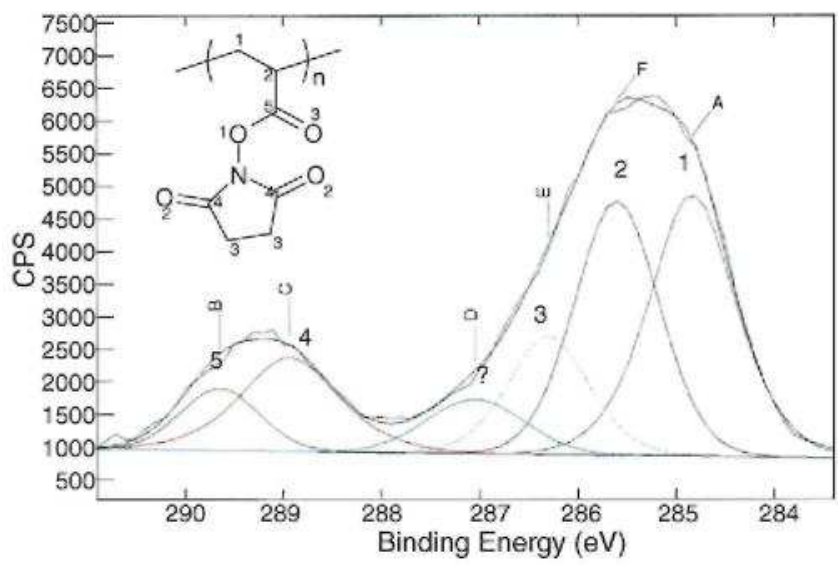

Fig. 4. XPS characterization of the electrografted PNSA coating (C1s area).

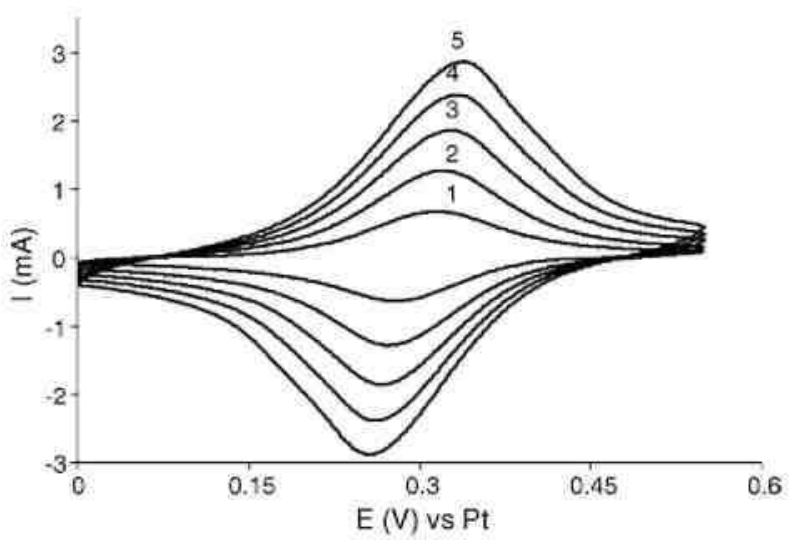

Fig. 5. Cyclic voltammogram recorded in ACN/TEAP for C-PNSA film after reaction with aminoferrocene at various scanning rates: (1) 50; (2) 100; (3) 150; (4) 200; and (5) $250 \mathrm{mV} / \mathrm{s}$.

First, polyethylene oxides end-capped with an acrylic function (A-PEO and A-PEO-A) have been successfully electro-grafted when dissolved in DMF/TEAP solution and reduced on a variety of substrates (metals, carbon, etc.). As shown in Fig. 6B and C, the ATR-FTIR analysis of the metallic surface after cathodic polarization, confirms the immobilization of the polymer coatings: the signals of the polyacrylic-PEO coatings (i.e. C-O-C ether at $1100 \mathrm{~cm}^{-1}, \mathrm{C}=\mathrm{O}$ ester at $1730 \mathrm{~cm}^{-1}$ ) have been clearly detected.

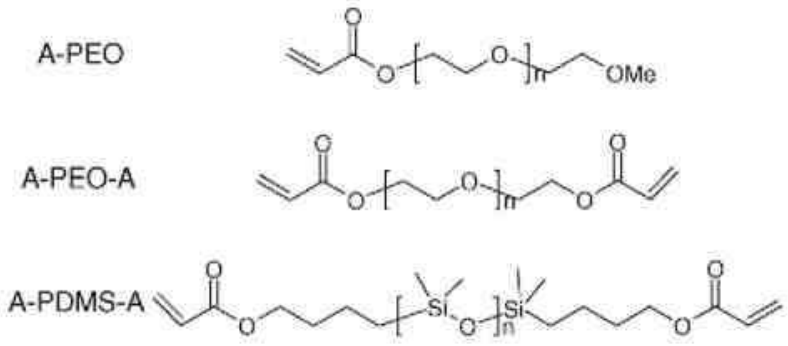

Scheme 6. Preformed reactive polymers used for the third approach. 


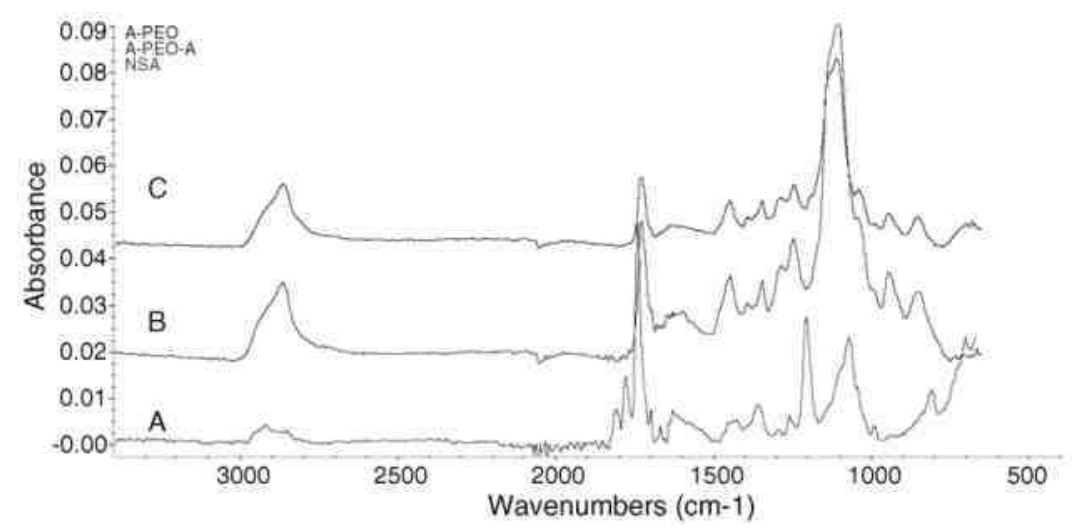

Fig. 6. Infrared spectra recorded on polished steel modified with (A) PNSA; (B) P(A-PEO-A); and (C) P(APEO).

\section{Table 1}

Contact angle of water on surfaces modified by electrografted macromonomers

\begin{tabular}{ll}
\hline Grafted film & Contact angle \\
\hline A-PEG & $25^{\circ} \pm 3^{\circ}$ \\
A-PEG-A Mn 258 & $48^{\circ} \pm 4^{\circ}$ \\
A-PEG-A Mn 700 & $<10^{\circ}$ \\
A-PDMS-A electrografted in DMF & $86^{\circ} \pm 3^{\circ}$ \\
A-PDMS-A electrografted in $\mathrm{CO}_{2}$ & $85^{\circ} \pm 3^{\circ}$ \\
\hline
\end{tabular}

The hydrophilicity of these coatings was established by contact angle measurements of water on the electrografted surfaces (Table 1). The highest hydrophilicity was observed for the difunctional A-PEO-A with the longer chain (Mn 700). In this case, the film hydrophilicity is very high (contact angle $<10^{\circ}$ ) due to the high content of PEO as compare to the acrylic backbone that limits the hydrophilicity in the two other cases. These highly hydrophilic coatings obtained in one-step from commercially available PEO derivatives are expected to be very efficient in preventing non-specific protein adsorption on metallic surfaces by inducing high hydrophilic character. These electro-generated films are thus highly promising in the field of implants coating.

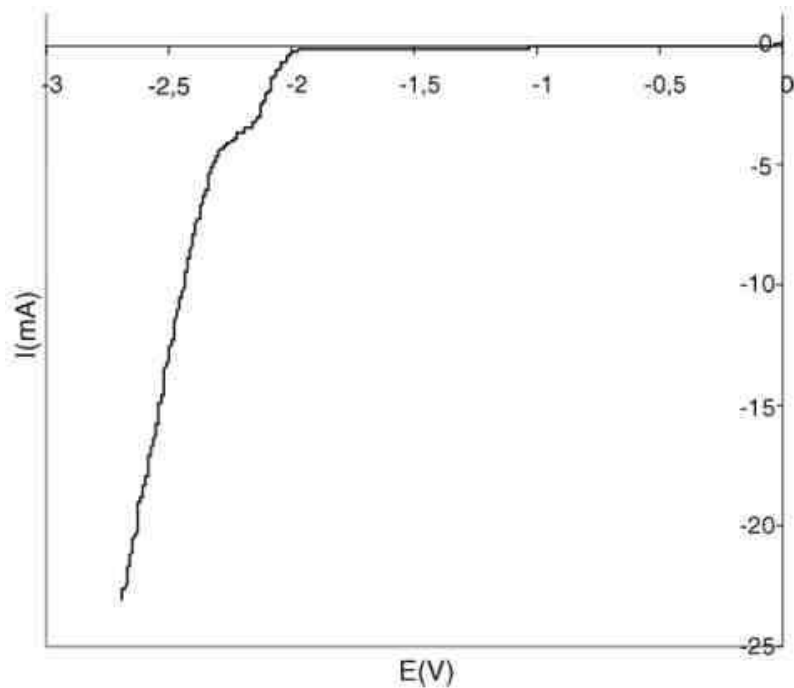

Fig. 7. Voltammogram for the electrografting of PDMS diacrylate in DMF/TEAP. 


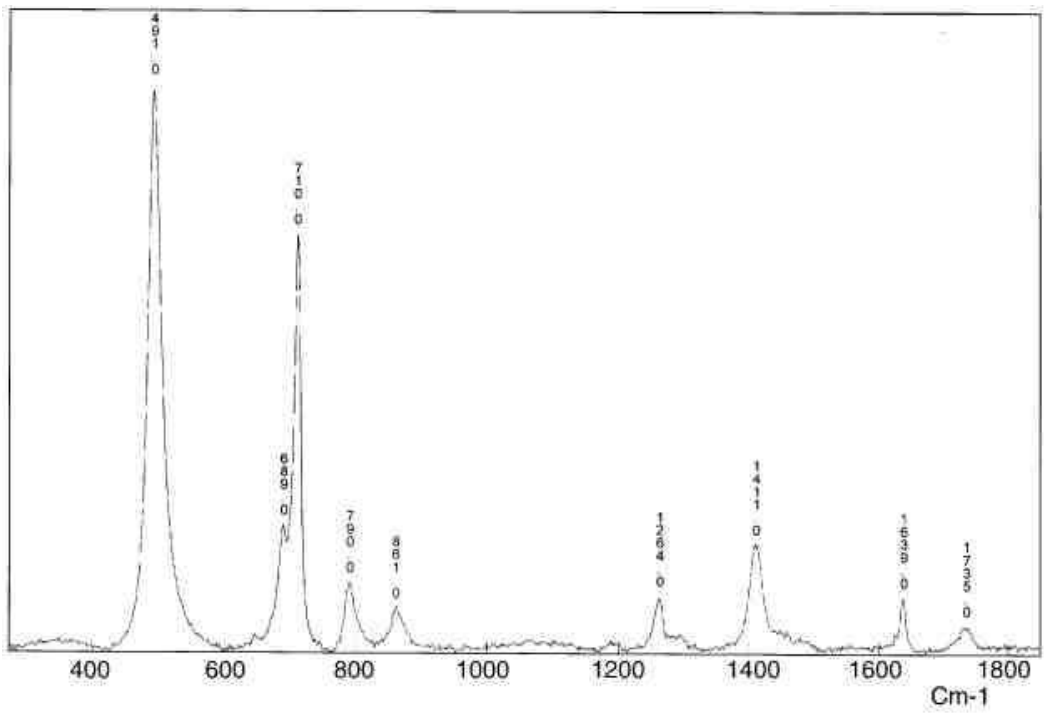

Fig. 8. Raman spectrum of the PDMS coating electrografted in DMF.

On the other hand, highly hydrophobic films have been prepared by direct electrografting of reactive polydimethylsilox-ane (A-PDMS-A, Scheme 6). Fig. 7 shows the voltammogram recorded for this macromonomer in DMF/TEAP. Even if the electrografting peak is not very well-defined in this case, the presence of the coating has been ascertained by Raman spectroscopy (Fig. 8). Strong bands at 490 and $710 \mathrm{~cm}^{-1}$ typical for Si-O bonds vibrations are clearly observed on the spectrum.

Interestingly enough, supercritical carbon dioxide has also been substituted for the usual organic solvent (DMF), which is quite toxic. This solvent is not very well-suited for electrochemistry because of low dissociation power of electrolytes. However, added with a minimum of DMF $(<10 \%)$, the dissolution of the conducting salt was successful, and the $\mathrm{CO}_{2}$-philic A-PDMS-A could be electrografted from this medium onto a carbon surface. Even if very preliminary, these results are thus quite promising. The use of this easily removable solvent is particularly interesting as far as PDMS coatings of biomedical devices are concerned.

The hydrophobicity of these coatings was ascertained by contact angle measurements (Table 1). Hydrophobic coatings are obtained even if the hydrophobicity is lower than a pure PDMS coating $\left(112^{\circ}\right)$. This is most probably due to the more polar acrylic component present in the coating. In this case, the other strategy consisting of combination of electrografting with the "grafting to" by hydrosilylation of PDMS end-capped with a silane on an electrografted poly(allylmethacrylate) film allows to reach much higher contact angle values.

\section{Conclusions}

Combination of electrografting and controlled/living polymerization ("grafting from" technique) has proved the ability of this two-step strategy in overcoming the major limitations of the electrografting process itself. Indeed, a larger range of organic polymer can be immobilized at the surface of electrically conducting substrates, as exemplified by polystyrene in this work. Moreover, new architectures can also be achieved such as diblock copolymers tethered to the conducting surface. Very importantly, the thickness of the films, which is limited at approximately $100 \mathrm{~nm}$ by electrografting, can be increased proportionally to the length of the polymer chains chemically grafted in the second step, thus in relation to the monomer to alkoxyamine ratio in the presented example (consistent with a controlled polymerization). This strategy can be extended to the large range of monomers that can be polymerized in a controlled manner by NMP with the purpose to tune the surface properties of glassy carbon, metals and steel while imparting adhesion to the organic coating.

The electrografting of the easily prepared $N$-succinimidyl acrylate is also a very efficient technique for imparting high reactivity to the surface of a variety of conducting substrates. This strongly adhering coating is a versatile anchoring film for a large series of nucleophiles, including aminoferrocene, enzymes, proteins and synthetic polymers. The grafting of ferrocene converts the PNSA film into an electroactive one, that could be used as mediator in electrochemical sensors. Grafting of enzymes and proteins, particularly onto QCM electrodes, makes the technique promising for devising biosensors.

Finally, the direct electrografting of preformed reactive polymers is a very promising strategy to extend further the potential of the electrografting technique. The control of the macromolecular parameters, such as molecular weight, of premade (co)polymers is a powerful tool for tuning the properties of strongly adhering organic films. In addition to the end-capped polymers presented here, random copolymers and (semi)telechelic polymers, block copolymers, star-shaped (co)polymers, dendrons, etc., of various molecular masses and compositions are 
candidates for chemisorption. Last but not least, this strategy is applicable not only to polymers prepared by a chain-growth mechanism but also to polycondensates (e.g., after derivatization of side or end-groups into acrylates).

These three strategies have thus proved efficiency in surface functionalization and in tuning the coatings properties.

\section{Acknowledgements}

The authors are much indebted to the "Belgian Science Policy" for general support under the auspices of the "Pôles d'Attraction Interuniversitaires: Supramolecular Chemistry and Supramolecular Catalysis". (PAI 5/3). C.J. is "Research Associate" près du Fonds National de la Recherche Scientifique (FNRS-Belgium). S.V. and H.S. are grateful to the "Fonds pour la Recherche en Industrie et Agriculture" (FRIA) for a fellowship.

\section{References}

[1] G. Cuny, J. Cao, J. Hauske, Tetrahedron Lett. 38 (1997) 5237.

[2] U. Velten, S. Tossati, R. Shelden, W. Caseri, U. Suter, Langmuir 15 (1999) 6940.

[3] N. Baute, P. Teyssié, L. Martinot, M. Mertens, P. Dubois, R. Jérôme, Eur. J. Inorg. Chem. (1998) 1711.

[4] N. Baute, C. Calberg, P. Dubois, C. Jérôme, R. Jérôme, L. Martinot, M. Mertens, P. Teyssié, Macromol. Symp. 134 (1998) 157.

[5] N. Baute, L. Martinot, R. Jérôme, J. Electroanal. Chem. 472 (1999) 83.

[6] N. Baute, C. Jérôme, L. Martinot, M. Mertens, V.M. Geskin, R. Lazza roni, J.L. Brédas, R. Jérôme, Eur. J. Inorg. Chem. (2001) 1097.

[7] C. Detrembleur, C. Jérôme, M. Claes, P. Louette, R. Jérôme, Angew. Chem. Int. Ed. Engl. 113 (7) (2001) 1308.

[8] S. Voccia, M. Claes, R. Jérôme, C. Jérôme, Macromol. Rapid Commun. 26 (10) (2005) 779-783.

[9] S. Voccia, L. Bech, B. Gilbert, R. Jérôme, C. Jérôme, Langmuir 20 (24) (2004) 10670-10678.

[10] M. Claes, S. Voccia, C. Detrembleur, C. Jérôme, B. Gilbert, Ph. Leclère, V.M. Geskin, R. Gouttebaron, M. Hecq, R. Lazzaroni, R. Jérôme, Macro molecules 36 (2003) 5926.

[11] M. Ignatova, S. Voccia, B. Gilbert, N. Markova, P.S. Mercuri, M. Galleni, V. Sciannamea, S. Lenoir, D. Cossement, R. Gouttebaron, R. Jérôme, C. Jérôme, Langmuir 20 (24) (2004) 10718-10726.

[12] C. Jérôme, S. Gabriel, S. Voccia, C. Detrembleur, M. Ignatova, R. Gout tebaron, R. Jérôme, J. Chem. Soc., Chem. Commun. 19 (2003) 2500.

[13] X. Lou, C. Jérôme, C. Detrembleur, R. Jérôme, Langmuir 18 (7) (2002) 2785-2788. 